GLOBAL CHILD HEALTH

\title{
The Programme for Global Paediatric Research
}

\author{
M Manley, A Zipursky
}

Arch Dis Child 2005;90:763-765. doi: 10.1136/adc.2004.068452

Millions of children die every year from malaria, tuberculosis, diarrhoea, neonatal disorders, and many other diseases found primarily in low and mid income countries. Many devoted clinicians and scientists have contributed significantly to the understanding and care of these diseases, however research remains disproportionately aimed at diseases affecting those in high income countries.

See end of article for authors' affiliations .....................

Correspondence to: Ms M Manley, The Programme for Global Paediatric Research, Dept of Paediatrics, The Hospital for Sick Children, Toronto, Ontario, Canada: margaret.manley@ sickkids.ca

Accepted 18 February 2005
$\mathrm{T}$ he disproportionate quantity of scientific research funds directed to the study of a small percentage of the world's disease burden has become known as the "10/90 gap". This concept, identified in 1990 by the Commission on Health Research for Development, referred to the fact that, at that time, only $10 \%$ of the money spent worldwide on health research was focused on the health problems of $90 \%$ of the global population. ${ }^{1}$ Children are, of course, profoundly affected by this gap.

The Programme for Global Paediatric Research (PGPR) is an international union of paediatric scientists and societies. It has been formed to address the disparity between the scientific resources available in high income countries and the quantity of scientific research aimed at child health in mid and low income countries. PGPR brings together scientists from throughout the world for the purposes of education, collaborative research, and ultimately the maintenance of child health and the cure of childhood

\section{GENESIS OF PGPR}

Pediatric Research is the official journal of the American Pediatric Society, the Society for Pediatric Research, the European Society of Pediatric Research, and the European Society of Paediatric Haematology and Immunology. During the years 1999-2003, when one of the authors (AZ) was the Editor-in-Chief of Pediatric Research, approximately 1100 articles were published in the journal, yet only four of those focused on malaria, tuberculosis, or severe malnutrition despite the major impact these diseases have on children in the developing world.

This observation led to an informal review of other major paediatric scientific journals and of the programme agendas for major paediatric clinical and research conferences. It became apparent that neither the journals nor the conferences had a strong focus on diseases diseases. primarily affecting children in the developing world.

A meeting to discuss this perceived problem was convened by AZ at the 2003 annual meeting of the US Pediatric Academic Societies (the American Pediatric Society, the Society for Pediatric Research, and the Ambulatory Pediatric Society) (PAS). Participants included the Presidents of the Japanese Pediatric Society, the International Pediatric Association, the American Pediatric Society, The Society for Pediatric Research, the Past-President of the Chinese Pediatric Society, representatives of Pediatric Research, USAID, and various other interested individuals.

At the meeting there was consensus that there is a great need to find ways to focus paediatric researchers on global health issues, facilitate communication between paediatric researchers throughout the world, and encourage collaborative study so that valuable contributions to global child health research can be made. In order to carry these ideas forward, PGPR was formed.

The Hospital for Sick Children in Toronto agreed to establish PGPR as a section within the Department of Paediatrics. This arrangement benefits the programme as it works to establish itself as a collaborative, international initiative, and it serves as an extension of the hospital's role in advancing international child health. Although the hospital supported the programme with "seed funds", ongoing funding of PGPR must come from external sources, through grants and donations. This is both an economic necessity and a principled response to the notion that all facets of participation in PGPR should be international.

\section{INAUGURAL SYMPOSIUM}

The first PGPR symposium was held during the 2004 PAS Annual Meeting.

The goal of that symposium was to introduce PGPR, bring major global child health issues to the large audience of researchers attending the PAS Meeting, in order to stimulate their interest in those issues, and begin dialogue about next steps. Symposium topics included Micronutrient Deficiency in Childhood; Malaria; and the Genetics of Tuberculosis.

\section{Micronutrient deficiency in childhood \\ Iron deficiency}

Symposium presentations began with a focus on iron deficiency, a major global health issue. Current research shows that besides causing anaemia, iron deficiency in infancy might permanently affect brain development.

Patricio Peirano (University of Chile, Santiago, Chile) described permanent changes in brain and 
behavioural development in children who had been iron deficient in infancy, but had recovered with iron therapy.

Matthew Burden (University of Michigan, Ann Arbor, USA) discussed his studies of 19 year old subjects who had been iron deficient in infancy. These individuals showed deficiencies in executive function. ${ }^{2}$

Cecilia Algarin (University of Chile, Santiago, Chile) described reduced executive and cognitive function in a group of 10 year old children who had been iron deficient in infancy. ${ }^{3}$

Adam Schmidt (University of Minnesota, Minneapolis, USA) presented studies of newborn rats whose mothers had been rendered iron deficient. This group showed differences in function and behaviour when compared with the control group. ${ }^{4}$

Thus, there is considerable evidence that iron deficiency in the fetus and newborn leads to interference in brain development and that these changes can be permanent. Since iron deficiency affects as many as $50 \%$ of children in Africa and Asia, ${ }^{5}$ it should be recognised as one of the world's significant health threats.

Stanley Zlotkin (The Hospital for Sick Children, Toronto, Canada) described the Micronutrient Sprinkles Programme, for which he is Director. This project is aimed at addressing the problem of iron deficiency in infants and children. While it is known that iron therapy in infancy will prevent iron deficiency, most forms of iron therapy for infants have major limitations. Iron fortified formulas can be effective; however, they can be costly and problematic in other ways, especially if used instead of breast milk. Iron supplement drops can also be effective, but taste, and therefore compliance, is a problem. As is the ever present risk of iron poisoning.

Dr Zlotkin and his group have developed a new way of providing iron therapy: "Sprinkles" are encapsulated micronutrients, packaged in single serving sachets. "Sprinkles" can be added to food without altering the food's smell, colour, or taste. In addition, Sprinkles are economical and simple to use. In addition to iron, various other micronutrients can be added to their formulations. These include zinc, vitamins A, C, and D, and folic acid. Dr Zlotkin reported that Sprinkles have been proven effective in the treatment of iron (and other vitamin) deficiencies in more than 2000 children in developing countries.

\section{Zinc deficiency}

Zulfiqar Bhutta (Aga Khan University, Karachi, Pakistan) and Robert Black (Johns Hopkins University, Baltimore, USA) presented information about the global problem of zinc deficiency.

It is estimated that one third of the world's population live in countries with a high risk of zinc deficiency; however diagnosis is difficult, so prevalence is not accurately known.

Dr Bhutta reviewed the significant impact of zinc deficiency on the immune system and zinc's importance for the maintenance of the intestinal mucosa. Both of these functions are relevant to the important clinical trials described by Dr Black.

Evidence of zinc deficiency and its importance can be ascertained from clinical trials of treatment and prevention.

Dr Black described zinc supplementation controlled trials in children in South-East Asia, Africa, Central and South America. These studies provided clear evidence of the importance of zinc supplementation for both the prevention and treatment of disease. Nine studies showed that prophylactic zinc administration reduced the incidence of diarrhoea; five studies showed a reduction in pneumonia; and two studies showed a significant reduction in malaria. Similarly zinc therapy substantially reduced the duration and severity of diarrhoea and pneumonia.

\section{Malaria}

Bernard Brabin (Liverpool School of Tropical Medicine, Liverpool, UK) discussed malaria as the most important preventable cause of low birth weight in many countries in Africa and also as a significant cause of fetal anaemia. Dr Brabin showed evidence that treating malaria during pregnancy significantly decreases the incidence and level of these complications.

Philip Fischer (Mayo Clinic, Rochester, USA) described the frequency with which malaria occurs in newborn infants, manifesting as anaemia and splenomegaly. Similar studies from the Republic of Côte d'Ivoire were reported by Douty Diomande (Teaching Hospital of Cocody Abidjan, Abidjan, Côte d'Ivoire). ${ }^{6}$

\section{Genetics of tuberculosis}

Erwin Schurr (McGill Centre for the Study of Host Resistance, Montreal, Canada) discussed the genetic basis of susceptibility to tuberculosis. He described the scope of the disease, with 8.4 million new cases each year, primarily in developing countries.

Genetic susceptibility to mycobacterial infections has been evidenced by animal studies and in humans by twin studies, as well as studies of morbidity and mortality following nontuberculous mycobacterial infections. Dr Schurr provided evidence that susceptibility to tuberculosis is a complex trait under multigene control. To date, five genes have been identified as being responsible for increased susceptibility to mycobacterial infections.

\section{INAUGURAL WORKSHOP}

Invited participants from 18 countries attended PGPR's first workshop, on the day after the symposium. The purpose of the workshop was to discuss the ongoing need for and potential of PGPR.

Five experts, each with experience in a different country or region, presented their perspectives on global child health research needs. These experts were: Demissie Habte (Consulting Health Specialist, Regional Human Development, Africa Technical Families, The World Bank, Washington, DC, USA); Hoosen M Coovadia (The Nelson Mandela School of Medicine, University of Natal, Congella, South Africa); Xiqiang Yang (Editor-in-Chief of the Chinese Journal of Pediatrics, Chongqing, China); José Ignacio Santos Preciado (Hospital Infantil de México Federico Gómez, Mexico City, Mexico); and Edward F Bell (University of Iowa, Iowa City, USA).

They also discussed their ideas of how PGPR could and should facilitate collaborative research and the establishment of sustainable research infrastructure in developing countries. Subsequent small group discussions focused on goals and plans for moving forward.

\section{OUTCOME OF INAUGURAL SYMPOSIUM AND WORKSHOP}

The symposium presentations brought PAS meeting attendees important information about major health issues affecting children in developing regions. Although the presented topics had not been previously considered at the meeting, many of the attendees had expertise that could significantly contribute to discussion and study of them. In addition, the attendees were very interested in the presentations. This was apparent from the large size of the audience (at times there was "standing room only") and from the strong positive feedback PGPR received from those who attended the symposium.

The workshop participants were enthusiastic, active, and productive, collectively supporting the programme and establishing clear recommendations for moving forward. 
Attendees suggested that PGPR continue to hold symposia at PAS meetings, as well as conduct meetings in other parts of the world. They also provided valuable ideas about topics for future symposia, workshops, programme structure and development, funding, partnerships, networking, and communication.

The involvement of invitees from low and middle income countries was one of the keys to the success of both the symposium and the workshop since PGPR is intended to be a truly international initiative, where global ideas are exchanged. Thus, PGPR provided travel funds for some attendees from low and middle income countries. External funding for this purpose was received from British Columbia Children's Hospital Foundation, March of Dimes, American Academy of Pediatrics, Section on International Child Health, Novartis Pharmaceuticals Canada Inc., and Pfizer Canada Inc. At the workshop, there was unanimous agreement that PGPR should continue to facilitate as much involvement by researchers and other experts from the developing world as possible.

\section{MOVING FORWARD}

Due to the success of the 2004 symposium and the interest it generated, another PGPR symposium will be held at the 2005 PAS Annual Meeting. With attendance of more than 5000, the PAS Meeting is an ideal opportunity to bring global health issues to the attention of scientists throughout the world.

One of the goals of PGPR is to facilitate the integration of global childhood health issues into the programmes of major paediatric research meetings around the world. Those meetings will serve as forums for prioritising, defining problems amenable to research, educating clinicians and scientists about what is being done and what needs to be done to improve the health of children in mid and low income countries, and collaborative planning of investigation and solutions.

Through the PGPR symposia at the PAS meeting, the above noted goal is beginning to be achieved. Recently, PGPR participated in a symposium on global paediatrics at the annual meeting of the European Society for Paediatric Research. It is hoped that this meeting will also continue to include PGPR and its focus on global child health issues.

In order to maximise the outcome from the resources available to it, and move towards accomplishing its goals, PGPR will initially use its workshops and symposia to focus on one issue at a time, developing a plan for action that will be applied to issues that are subsequently chosen.

The current plan for action involves:

- Examining the inherent and surrounding issues of the chosen topic and identifying the character and extent of it as a global health problem. This step includes combining pathophysiology with clinical observations and sharing data on an international scale.

- Establishing research priorities within the chosen topic. These priorities can and should relate to prevention, treatment, and follow up and must include study and discussion regarding country or region specific appropriate research methodology.

- Facilitating education and collaboration surrounding the chosen topic.

- Developing a global network of researchers for communicating information about the chosen topic.

PGPR's first focus topic is birth asphyxia. The next PGPR symposium (to be held at the 2005 PAS Meeting) is entitled "Global Perspectives on Birth Asphyxia". That theme will follow through to a PGPR workshop the next day.

Birth asphyxia has been chosen as the first focus topic because:

- It is a major child health issue, responsible for over one million deaths annually

- Studying birth asphyxia supports the millennium development goal of reducing by two thirds the mortality rate among children under 5 by the year 2015

- This topic is an ideal one to bring to the PAS Meeting, since that conference provides the opportunity to attract widespread, expert participation in the symposium and the workshop.

\section{CONCLUSION}

PGPR has been successfully established as a consortium of societies, clinicians, and scientists involved in paediatric research. Its overarching goal is to mobilise the resources and expertise of this international community of researchers for the study and cure of diseases that affect so many children throughout the world.

\section{Authors' affiliations}

M Manley, A Zipursky, The Programme for Global Paediatric Research, Dept of Paediatrics, The Hospital for Sick Children, Toronto, Ontario, Canada

Funding: External travel support of some symposium and workshop attendees from mid and low income countries was received from British Columbia Children's Hospital Foundation, March of Dimes, American Academy of Pediatrics, Section on International Child Health, Novartis pharmaceuticals Canada Inc, and Pfizer Canada Inc.

Competing interests: none declared

\section{REFERENCES}

1 Global forum for health research. 10/90 report on health research 2003 2004. http://www.globalforumhealth.org/pages/index.asp (accessed 1 November 2004).

2 Burden M, Koss, M, Lozoff B, et al. Neurocognitive differences in 19-year olds treated for iron deficiency in infancy. Pediatr Res 2004;55:279A.

3 Algarin CR, Peirano PD, Just $E$, et al. Cerebral executive function in preadolescents is affected by iron deficiency in infancy. Pediatr Res 2004:55:279A.

4 Schmidt AT, Waldow KJ, Salinas JA, et al. The long-term behavioral effects of fetal/neonatal iron deficiency on a hippocampally dependent learning task in the rat. Pediatr Res 2004;55:279A.

5 Foege W. Keynote address: Issues in overcoming iron deficiency. J Nutr 2002;132:790S-3S

6 Diomande D, Folquet-Amorissani M, Dainguy M-e, et al. Malaria among newborn with infection risk. Pediatr Res 2004;55:280A. 